\title{
A Three-step Method for Three-way Clustering by Similarity-based Sample's Stability
}

\author{
Jin Zhu, ${ }^{1}$ Dongqin Jiang, ${ }^{2}$ and Pingxin Wang ${ }^{1}{ }^{1}$ \\ ${ }^{1}$ School of Science, Jiangsu University of Science and Technology, Zhenjiang, Jiangsu, 212003, China \\ ${ }^{2}$ School of Computer, Jiangsu University of Science and Technology, Zhenjiang, Jiangsu, 212003, China \\ Correspondence should be addressed to Pingxin Wang; pingxin_wang@hotmail.com
}

Received 6 September 2021; Accepted 17 January 2022; Published 27 February 2022

Academic Editor: Jian Lin

Copyright ( 12022 Jin Zhu et al. This is an open access article distributed under the Creative Commons Attribution License, which permits unrestricted use, distribution, and reproduction in any medium, provided the original work is properly cited.

Clustering is an important research field in machine learning. Traditional clustering approaches are not very effective in dealing with clusters having overlapping regions. To better capture the three types of relationships between a cluster and a sample, namely, belong-to fully, belong-to partially and not belong-to fully, we propose a theory of similarity-based sample's stability and develop a three-step method for three-way clustering by integrating similarity-based sample's stability into the idea of three-way clustering in this paper. In the proposed theory, the similarity of two samples is used to define the frequencies of two samples and the samples stability is calculated based on the defined frequencies and determinacy function. With this stability, the universe is divided into stable set and unstable set. The samples in the stable set are assigned into the core region of each cluster by using traditional clustering algorithm. The samples in the unstable set are assigned into the fringe region of corresponding cluster according to distances between the elements and the centers of the cluster core regions. Therefore, a three-way clustering is naturally formed. Experimental results on datasets show that this method can improve the structure of the clustering results.

\section{Introduction}

Data clustering is one of the most fundamental topics for data exploration in machine learning and plays has an important role in many fields such as information granulation, image analysis, network structure analysis and others [1-4]. The purpose of clustering is to discover the underlying structure of a data set by organizing the samples in the data set into several clusters such that the objects within a cluster are highly similar but remarkably dissimilar with objects in other clusters [5]. many researchers have done a series of research on clustering problem in the past decades and various kinds of clustering algorithms have been developed in the literature, including partitional, hierarchical, densitybased and grid-based clustering and so on. A comprehensive review of clustering algorithms can be found in [6].

According to the relation between the samples and the clusters, the existing algorithms for clustering can be divided into two categories: hard clustering and soft clustering. Hard clustering methods, such as k-means [7], spectral clustering
[8], represent a cluster by a set with a crisp boundary, where the objects in the set belong to this cluster definitely and the objects not in the set do not belong to this cluster definitely. Only two relations between the samples and the clusters are considered in the process of clustering, i.e, belong-to and not belong to. We use an example $V$ with 6 samples to illustrate this phenomenon in Figure 1. The samples in data set $V$ are grouped into disjoint cluster $C_{1}$ and cluster $C_{2} . C_{1}$ divides the universe into two sets, i.e., the set inside $C_{1}$ and the set outside $C_{1}$. The samples inside $C_{1}$ belong to the cluster and the samples outside $C_{1}$ do not belong to the cluster. The same is true about $C_{2}$.

Hard clustering methods ignore the possibility that a sample can belong to more than one cluster and lead to some exhaustive and exclusive clusters representing the data. Although the requirement that each cluster must be represented by a set with clear boundary leads to easy analytical results, assigning uncertain elements into a cluster may increase decision risk. In order to relax the constraint, many soft clustering methods were proposed for different 

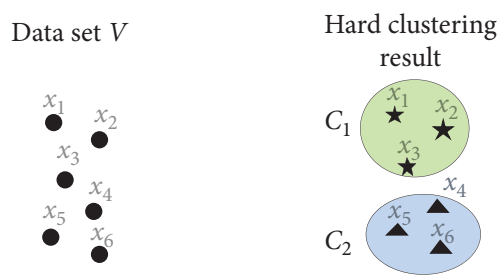

FIGURE 1: An example of hard clustering results.

application backgrounds [9-12]. As one of the effective tools to deal with uncertainty, three-way decision [13] trisects a universe and represents the uncertainty in the form of noncommitment. The main idea of three-way decision is thinking in threes, which is consistent with human's cognitions to solve the problem in the real world [14]. Many soft computing models for learning uncertain concepts, such as rough sets [15], fuzzy sets [16] and shadowed sets [17], can be reinvestigated within the framework of three-way decision [14].

By incorporating three-way decision with clustering analysis, $\mathrm{Yu}$ [18-20] proposes another model of soft clustering, i.e., three-way clustering. Different from the crisp representation of a cluster, three-way clustering uses the core region (Co), the fringe region ( $\mathrm{Fr}$ ), and the trivial region ( $\mathrm{Tr}$ ) to represent a cluster, which $\mathrm{Tr}$ can be expressed as the complement of the union of Co and Fr. The samples that belong to the cluster definitely are assigned to the Co and the samples that do not belong to the cluster definitely are assigned to the Tr. For the samples with uncertainty or information insufficient, we assign them into the Fr to give the further decision after obtaining more information. The above process reflects the three types of relationship between an object and a cluster, namely, belong-to definitely, uncertain and not belong-to definitely. Figure 2 shows one possible cluster obtained by three-way clustering method.

Three-way clustering uses the core region and the fringe to represent a cluster. How to develop different strategies to construct the core region and the fringe region has become the key problem of three-way clustering. In recent years, three-way clustering has attracted a lot of research, and many three-way clustering algorithm were obtained [21-27]. Recently, by observing that the samples can change between clusters in different clustering results, Li et al. [28] introduced the concept of sample's stability based on the coassociation frequencies in a clustering ensemble problem and presented a clustering ensemble algorithm based on sample's stability. Sample's stability is to quantify the contributions to detection of the clustering structure. The samples with higher stabilities can be used to discover the core region of three-way cluster, while the samples with lower stabilities are assigned into the fringe regions of corresponding clusters. The idea gives a new method for three-way clustering.

There are many different methods to define the sample's stability. In this paper, we develop a new three-way clustering algorithm by similarity-based sample's stability. In the proposed method, we use the similarity of two samples to define the co-association frequency and compute the

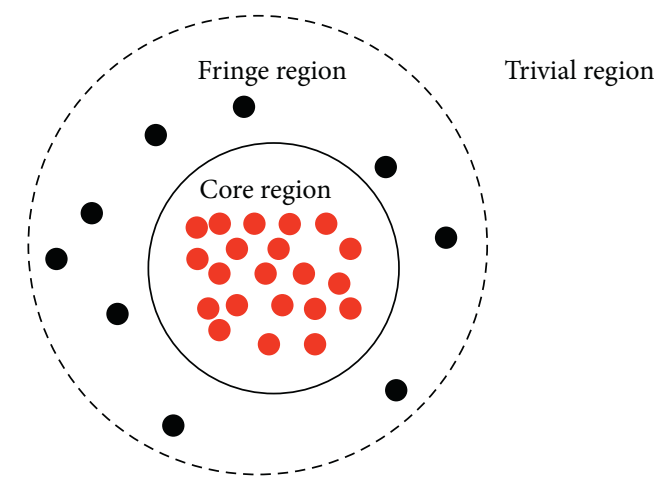

Figure 2: A demonstration of three-way cluster.

sample's stability based on the defined frequencies. All samples are divided into stable set and unstable set based on sample's stability. The stable set can be used to find the prestructure of the universe, which provides instructive information to the assignments of the samples in the unstable set. Concretely, the samples in the stable set are assigned into the core region of each cluster by using traditional clustering algorithm. The samples in the unstable set are assigned into the fringe region of corresponding cluster according to distances between the elements and the centers of the cluster core region. Therefore, a three-way clustering is naturally formed.

In comparison with the existing research, the main contributions of our work are as follows:

- Base on the similarity of two samples, a new definition of co-association frequencies is proposed and the relation between the sample's stability is discussed.

- A novel three-way clustering algorithm by similaritybased sample's stability is proposed. The samples with higher stabilities are assigned into the core region of each cluster by $\mathrm{k}$-means and the samples with lower stabilities are assigned into the fringe region of corresponding cluster according to distances between the elements and the centers of the cluster core region.

- The similarity-based sample's stability that is measured by the proposed method is verified by experiments on UCI data sets. Six UCI data sets are used to show the effectiveness of the proposed three-way clustering algorithm.

The rest of this paper is organized as follows. In Section 2, we review the concept of sample's stability and propose the theory of similarity-based sample's stability . A new three-way clustering algorithm by using similarity-based sample's stability is proposed in Section 3. Section 4 shows experimental results based on a bunch of public standard data sets.

\section{Similarity-Based Sample's Stability}

Sample's stability was first introduced by Li et al. [28] based on the co-association frequencies in a clustering ensemble problem. Giving a base clustering results of data set $X=$ $\left\{x_{1}, x_{2}, \ldots x_{n}\right\}$, the relationship of any two samples has three 
cases. The first case is that they are assigned to the same cluster in all clustering results and the second case is that they are assigned to different clusters in all clustering results. The last case is that they are assigned to the same cluster in some clustering results. The frequency of two samples appeared in the same cluster are defined as the co-association frequency of two samples. For the first case and the second case, the co-association frequency of two samples equals 1 and 0 , respectively. Regardless of the first case or the second case, the relationship between two samples is certain. For the case 3, the co-association frequency of two samples is in $(0,1)$ and the relationship between them is unstable.
Based on the distribution of the co-association frequencies, we can obtain a threshold $t$ to by Otsu algorithm [29] to represent the most unstable frequency.

The co-association frequency of two samples describes the probability of assigning into the same cluster. There are different methods to define the co-association frequency of two samples. In this paper, we adopt the similarity in spectral clustering [30] to represent the co-association frequency. As we know, there are three kinds of methods to define the similarity in spectral clustering. Here, we use the following one.

$$
p_{i j}=\left\{\begin{array}{l}
0, \text { if } \quad x_{i} \notin \mathrm{KNN}\left(x_{j}\right) \quad \text { or } \quad x_{j} \notin \mathrm{KNN}\left(x_{i}\right), \\
\exp \left(-\frac{d^{2}\left(x_{i}, x_{j}\right)}{2 \sigma^{2}}\right), \text { if } \quad x_{i} \in \operatorname{KNN}\left(x_{j}\right) \text { and } x_{j} \in \operatorname{KNN}\left(x_{i}\right),
\end{array}\right.
$$

where, $\mathrm{KNN}\left(x_{i}\right)$ represents the $K$ nearest neighbor of $x_{i}$, i.e., $\mathrm{KNN}\left(x_{i}\right)$ satisfies,

- $\mathrm{KNN}\left(x_{i}\right) \subset X$;

- $\left|\mathrm{KNN}\left(x_{i}\right)\right|=K$;

- $\forall x_{j} \in \mathrm{KNN}\left(x_{i}\right), x_{k} \notin \mathrm{KNN}\left(x_{i}\right) \mid=K$, then, dist $\left(x_{i}, x_{j}\right) \leq \operatorname{dist}\left(x_{i}, x_{k}\right)$.

Because a higher vale and a lower value of the co-association frequency mean a more stable relation between two samples $x_{i}$ and $x_{j}$, we can not use the co-association frequency to represent the stable level directly. We should design a function in $[0,1]$, which have high values at both ends of $[0,1]$ but low value at the most unstable frequency. This kind of function is named as the determinacy function, which is defined as follows:

Definition 1 (Determinacy function) [28]: $f$ is a determinacy function if for arguments $p \in[0,1]$ and with a parameter $t \in(0,1)$ it satisfies:

(1) If $p<t, f(p)<0$; if $p>t, f^{\prime}(p)>0$.

(2) If $p_{i}<t<p_{j}$, and $\left(t-p_{i} / p_{j}-t\right)=(t / 1-t)$, $f\left(p_{i}\right)=f\left(p_{j}\right)$.

From the definition, we can find that the determinacy function is monotonically decreasing function when $p<t$ and monotonically decreasing function when $p>t$. The function obtains the minimum value on $t$. Another properties is that the determinacy function is unbiased on both sides of $t$. From the first condition of Definition 1, we can obtain that for any two point $p_{i}$ and $p_{j}$ are located on the same side of $t$, the farther possibility point will have a higher determinacy value. From the second condition of Definition 1 , we can conclude that for any two point $p_{i}$ and $p_{j}$ are located on the different sides of $t, f\left(p_{i}\right)>f\left(p_{j}\right)$ if $p_{i}$ and $p_{j}$ satisfy $p_{i}<t<p_{j}$ and $(1-t)\left(t-p_{i}\right)>t\left(p_{j}-t\right)$.

From Definition 1, there are many different mathematical functions satisfying the conditions of determinacy function. Here, we give two examples as following.

$$
\begin{aligned}
& f_{l}\left(p_{i j}\right)= \begin{cases}\left|\frac{p_{i j}-t}{t}\right|, & p_{i j}<t \\
\left|\frac{p_{i j}-t}{1-t}\right|, & p_{i j} \geq t\end{cases} \\
& f_{q}\left(p_{i j}\right)= \begin{cases}\left(\frac{p_{i j}-t}{t}\right)^{2}, & p_{i j}<t \\
\left(\frac{p_{i j}-t}{1-t}\right)^{2}, & p_{i j} \geq t\end{cases}
\end{aligned}
$$

$f_{l}\left(p_{i j}\right)$ is called as a linear determinacy function and $f_{q}\left(p_{i j}\right)$ is called as a quadratic determinacy function, respectively. In Equation (2) and (3), the threshold $t$ is learned by using Otsu algorithm [29]. The idea of Otsu algorithm is to solve a linear discriminant problem. Suppose $D=\left\{d_{1}, d_{2}, \ldots, d_{m}\right\}$ is a vector with $m$ elements and $D$ is divided into two groups $g_{0}$ and $g_{1}$ as following,

$$
\begin{aligned}
& g_{0}=\left\{d_{i}, d_{i}<t_{0}, 1 \leqslant i \leqslant m .\right\} \\
& g_{1}=\left\{d_{i}, d_{i} \geqslant t_{0}, 1 \leqslant i \leqslant m .\right\} .
\end{aligned}
$$

The goal of Ostu algorithm is to search the threshold $t^{*}$ to maximize the following between-class variance $\sigma_{t}$,

$$
\sigma_{t}=w_{0}\left(\mu_{0}-\mu\right)^{2}+w_{1}\left(\mu_{1}-\mu\right)^{2},
$$

where 


$$
\begin{aligned}
& w_{0}=\frac{\left|g_{0}\right|}{|D|}, w_{1}=\frac{\left|g_{1}\right|}{|D|}, \\
& \mu_{0}=\frac{\sum_{d_{i} \in g_{0}} d_{i}}{\left|g_{0}\right|}, \mu_{1}=\frac{\sum_{d_{i} \in g_{1}} d_{i}}{\left|g_{1}\right|}, \mu=\frac{\sum_{d_{i} \in D} d_{i}}{|D|} .
\end{aligned}
$$

The following Algorithm 1 is the process of Otsu [29]. Determinacy function projects the co-association frequency into a relationship stability space. We can use the values of determinacy function to quantify the stability of a sample. Giving a determinacy function $f$, we can define the stability of a sample $x_{i}$ as following.

Definition 2 (Sample's stability) [28]: Suppose $f$ is a determinacy function. The stability of $x_{i}, s\left(x_{i}\right)$, is calculated by:

$$
s\left(x_{i}\right)=\frac{1}{n} \sum_{j=1}^{n} f\left(p_{i j}\right) \text {. }
$$

Next, we use an example to address the process of computing sample's stability. Suppose that $X=\left\{x_{1}, x_{2}, x_{3}, x_{4}, x_{5}, x_{6}\right\}$ is a data set with 6 samples. The distances of $x_{i}$ and $x_{j}$ are listed as Table 1 .

In order to compute the co-association frequency by equation (1), we set the size of neighbor $K=3$ and parameter $\sigma=1$. From Table 1 , we have $\operatorname{KNN}\left(x_{1}\right)=$ $\left\{x_{1}, x_{2}, x_{3}\right\}, \operatorname{KNN}\left(x_{2}\right)=\left\{x_{1}, x_{2}, x_{3}\right\} \quad$ and $\operatorname{KNN}\left(x_{3}\right)=$ $\left\{x_{2}, x_{3}, x_{4}\right\}$. Because $x_{1} \in \operatorname{KNN}\left(x_{2}\right)$ and $x_{2} \in K N N\left(x_{1}\right)$, we get the co-association frequency $p_{12}=e^{-1 / 2}$. Similarly, $p_{13}=0$ because $x_{1} \notin \mathrm{KNN}\left(x_{3}\right)$. Repeating the above process for other samples, we can obtain all the co-association frequencies. All the pairwise frequencies will form a $6 \times 6$ matrix as Table 2.

Based on the co-association matrix of, the most unstable frequency can be learned $t=0.4647$ by Otsu algorithm. According to the Definition 2, we get the sample's stabilities of $X$ by using linear determinacy function $f_{l}\left(p_{i j}\right)$,

$$
\begin{aligned}
& s\left(x_{1}\right)=0.8775 ; s\left(x_{2}\right)=0.7611 ; s\left(x_{3}\right)=0.7611 ; \\
& s\left(x_{4}\right)=0.7064 ; s\left(x_{5}\right)=0.7611 ; s\left(x_{6}\right)=0.8350 .
\end{aligned}
$$

\section{Three-Step Method for Three-Way Clustering by Similarity-Based Sample's Stability}

According to the discussion in previous section, we can calculate the stability of each sample in a data set. With these stability values, the universe can be divided into two sets through a threshold, i.e., the set with higher stability values and the set with lower stability values. For the samples with higher stability values, they have robust relationships with other samples and can be assigned to certain cluster in the process of clustering. For the samples with lower stability values, they do not have a clear relationships with other samples and assigning them into a certain cluster will increase decision risk.

Three-way clustering was proposed by $\mathrm{Yu}$ et al. [18-20], which uses the coreregion (Co), the fringe region (Fr), and

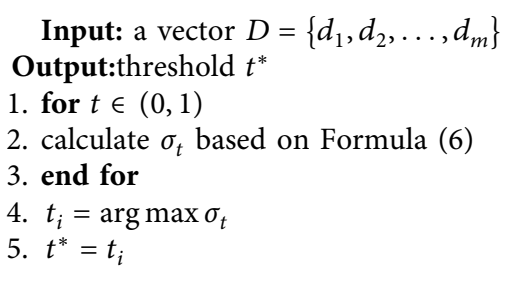

Algorithm 1: Otsu

TABle 1: The sample's distances of $X$

\begin{tabular}{lcccccc}
\hline distance & $x_{1}$ & $x_{2}$ & $x_{3}$ & $x_{4}$ & $x_{5}$ & $x_{6}$ \\
\hline$x_{1}$ & 0 & 1 & 2 & 3 & 4 & 5 \\
$x_{2}$ & 1 & 0 & 1.5 & 2.5 & 3.5 & 4.5 \\
$x_{3}$ & 2 & 1.5 & 0 & 1 & 2.5 & 3 \\
$x_{4}$ & 3 & 2.5 & 1 & 0 & 1 & 2 \\
$x_{5}$ & 4 & 3.5 & 2.5 & 1 & 0 & 1.5 \\
$x_{6}$ & 5 & 4.5 & 3 & 2 & 1.5 & 0 \\
\hline
\end{tabular}

the trivial region ( $\mathrm{Tr}$ ) to represent a cluster, which $\mathrm{Tr}$ can be expressed as the complement of the union of Co and Fr. Since $\mathrm{Tr}$ is the complement of the union of Co and Fr, threeway clustering represents a three-way cluster $C_{i}$ as

$$
C_{i}=\left(\operatorname{Co}\left(C_{i}\right), \operatorname{Fr}\left(C_{i}\right)\right)
$$

The result of three-way clustering can be represented as

$$
\begin{aligned}
\mathbb{C}= & \left\{\left(\operatorname{Co}\left(C_{1}\right), \operatorname{Fr}\left(C_{1}\right)\right),\left(\operatorname{Co}\left(C_{2}\right), \operatorname{Fr}\left(C_{2}\right)\right),\right. \\
& \left.\ldots,\left(\operatorname{Co}\left(C_{k}\right), \operatorname{Fr}\left(C_{k}\right)\right)\right\},
\end{aligned}
$$

where subsets $\operatorname{Co}\left(C_{i}\right), \operatorname{Fr}\left(C_{i}\right)$ have the following properties.

$$
\begin{aligned}
\operatorname{Tr}\left(C_{i}\right) \cup \operatorname{Co}\left(C_{i}\right) \cup \operatorname{Fr}\left(C_{i}\right) & =U, \\
* & =\phi, \\
\operatorname{Co}\left(C_{i}\right) \cap \operatorname{Fr}\left(C_{i}\right) & =\phi \\
* & \\
\operatorname{Co}\left(C_{i}\right) \cap \operatorname{Tr}\left(C_{i}\right) & =\phi, \\
* & \\
\operatorname{Fr}\left(C_{i}\right) \cap \operatorname{Tr}\left(C_{i}\right) & =\phi .
\end{aligned}
$$

Motivated by three-way clustering, this paper presents a new three-way clustering algorithm by similarity-based sample's stability (3WC-SSS for short). The main idea of $3 \mathrm{WC}$-SSS is dividing the universe into stable set and unstable set according to the similarity-based sample's stability and handling the samples in stable set and unstable set differently.

3.1. Step 1: Dividing the Universe into a Stable Set and an Unstable Set. In the proposed algorithm, the stability of each sample is first computed by using definition of similaritybased sample's stability in the previous section and the universe is divided into two sets through a threshold, i.e., the set with higher stability values and the set with lower stability 
TABle 2: The co-association matrix of $X$

\begin{tabular}{lcccccc}
\hline frequency & $x_{1}$ & $x_{2}$ & $x_{3}$ & $x_{4}$ & $x_{5}$ & \\
\hline$x_{1}$ & 1 & $e^{-1 / 2}$ & 0 & 0 & 0 & 0 \\
$x_{2}$ & $e^{-1 / 2}$ & 1 & $e^{-9 / 8}$ & 0 & 0 & 0 \\
$x_{3}$ & 0 & $e^{-9 / 8}$ & 1 & $e^{-1 / 2}$ & 0 \\
$x_{4}$ & 0 & 0 & $e^{-1 / 2}$ & $e^{-1 / 2}$ & $e^{-1 / 2}$ & $e^{-2}$ \\
$x_{5}$ & 0 & 0 & 0 & $e^{-2}$ & $e^{-9 / 8}$ \\
$x_{6}$ & 0 & 0 & 0 & & $e^{-9 / 8}$ \\
\hline
\end{tabular}

values. The group with higher stability is defined as the stable set while the group with lower stability is defined as the unstable set. For the samples in the stable set, they have robust relationships with other samples, which can be assigned into the core region of each cluster by using $\mathrm{k}$-means. After obtaining the core region of each cluster $\operatorname{Co}\left(C_{1}\right), \operatorname{Co}\left(C_{2}\right), \ldots, \operatorname{Co}\left(C_{k}\right)$, the next problem is to assign the samples in the unstable set to the fringe region of each cluster. We compute its local co-association coefficient corresponding to $\operatorname{Co}\left(C_{1}\right), \operatorname{Co}\left(C_{2}\right), \ldots, \operatorname{Co}\left(C_{k}\right)$, respectively, and assign the sample to $\operatorname{Fr}\left(C_{i}\right)$ according to its local stability. The workflow of the proposed 3WC-SSS algorithm can be depicted by Fig 3 .

Three-way clustering uses the core region (Co), the fringe region ( $\mathrm{Fr}$ ), and the trivial region ( $\mathrm{Tr}$ ) to represent a cluster. Since $\mathrm{Tr}$ can be expressed as the complement of the union of Co and Fr, we can represent a three-way cluster by a pair of the set of core objects and the set of boundary or fringe objects. The representation of three-way clustering by using three regions or, equivalently, a pair of sets to represent a cluster chapters the three types of relationships between an element and a cluster, namely, belong-to definitely, not belong-to definitely, and uncertain. One of the main tasks of three-way clustering is to construct the core region and the fringe region of each cluster. The strategy of 3WC-SSS algorithm is dividing the universe into universe core and universe fringe by using similarity-based sample's stability and assigning the samples in universe core and the universe fringe to the core region and the fringe region of each cluster, respectively. So, the first step of 3WC-SSS algorithm is to obtain the sample's stability based on Definition 2. Suppose $X=\left\{x_{1}, \ldots, x_{n}\right\}$ is a data set with $n$ samples. Firstly, we use equation (1) to get co-association frequency $p_{i j}$ of $x_{i}$ and $x_{j}$, where the size $K$ of neighbour is an important parameter. Too large and too small value of $K$ are not appropriate. Too large $K$ will increase the costs of computation and too small $K$ will lead to local problem. In this paper, we set $K=[n /(2 k)], k$ is the number of the clusters.

In this paper, we use the linear determinacy function $f_{l}(x)$ to compute the sample's stability. After we get the coassociation matrix, the stabilities of all samples $S=\left\{s\left(x_{1}\right), s\left(x_{2}\right), \ldots, s\left(x_{n}\right)\right\}$ can be obtained by Definition 2. Similarly, taking $S=\left\{s\left(x_{1}\right), s\left(x_{2}\right), \ldots, s\left(x_{n}\right)\right\}$ as an input, we can use Otsu algorithm to obtain a threshold $s$ and the data set $X$ is divided into two crisp sets according to threshold. We name these two groups as the stable set and the unstable set, respectively, which are given by:

$$
\mathrm{ST}(X)=\left\{x_{i} \mid s\left(x_{i}\right)>s\right\}
$$

and

$$
\mathrm{US}(X)=\left\{x_{i} \mid s\left(x_{i}\right) \leq s\right\}
$$

3.2. Step 2: Finding the Core Region of Each Cluster. The samples in the stable set have higher stability, which means that the samples have robust relationships with other samples. Because the samples in the core region belong to the cluster definitely, they should have robust relationships with other samples. So, we assign the the samples of the stable set into the core region of each cluster in our proposed 3WC-SSS. All the hard clustering algorithm can be used to divide the stable set into $k$ clusters. This paper adopts k-means [7] algorithm to realize this result. The main idea of $\mathrm{k}$-means is to randomly select $k$ initial centers, then, cluster the samples $k$ groups according to the shortest distance to the center points, update the centers by means of the data in the cluster until the center no longer changes. K-means algorithm aims at minimizing the following objective function,

$$
J=\min \sum_{j=1}^{k} \sum_{x_{i} \in C_{j}}\left\|v_{i}-\mu_{j}\right\|^{2},
$$

where $\left\|x_{i}-\mu_{j}\right\|$ represents the distance between $x_{i}$ and the centre $\mu_{j}$ of $j$-th cluster.

Based on the above discussions, we get the core region of each cluster by assigning the samples with higher stability into several clusters through $\mathrm{k}$-means. The procedure of obtaining core region of each cluster can be described as Algorithm 2.

\subsection{Step 3: Finding the Fringe Region of Each Cluster.} Another task of 3WC-SSS is to get the fringe region of each cluster. For the samples in the unstable set, it is reasonable to assign them into the fringe region of the corresponding cluster because they do not have a clear relationships with other samples. Since the core regions have been obtained by stable set, the relationship between the samples of unstable set and the discovered core regions can be used to decide the fringe region of each cluster. In this paper, we introduce the definition of local co-association coefficient and use this definition to get the fringe region. The definition of local coassociation coefficient is as following. 


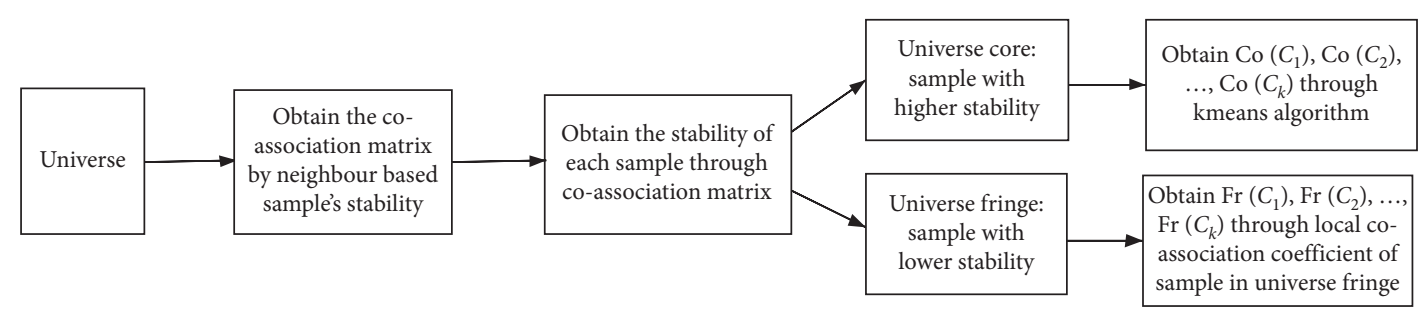

FIgURE 3: The workflow of 3WC-SSS algorithm.

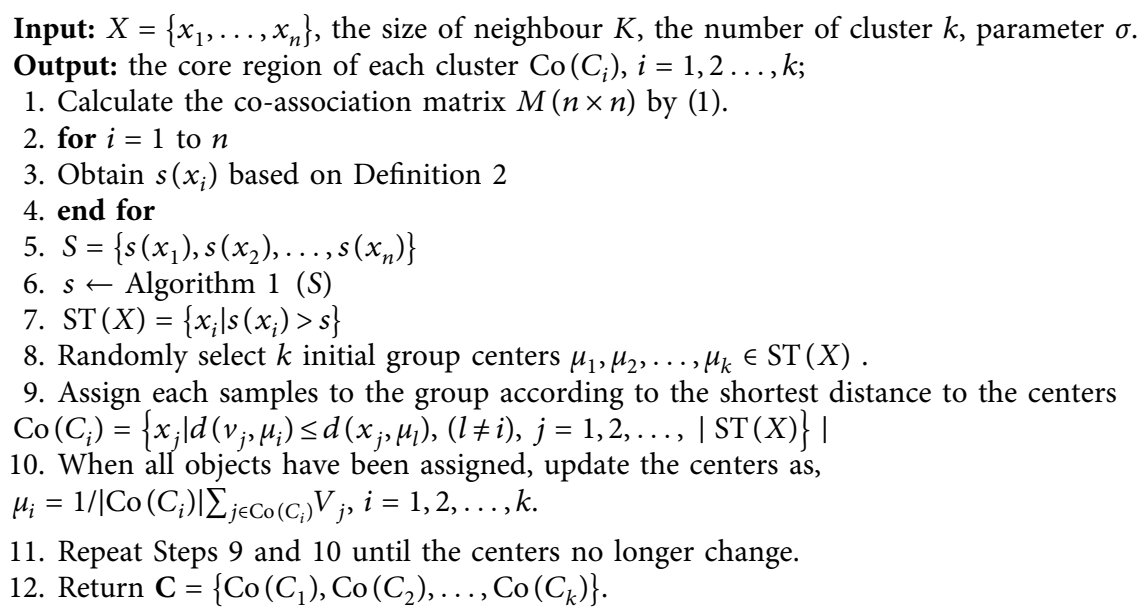

Algorithm 2: Finding the core region of each cluster

Definition 3. Suppose $X$ is a date set, $X_{1}$ is subset of $X, p_{i j}$ is the co-association frequency between two samples $x_{i}$ and $x_{j}$. The local co-association coefficient $A_{l}\left(x_{i}\right)$ between a sample $x_{i}$ and subset $X_{1}$ is defined as following,

$$
A_{l}\left(x_{i}\right)=\sum_{x_{j} \in X_{1}} p_{i j} \text {. }
$$

The local co-association coefficient reflects the affiliation between the sample and the subset. The higher the value, the closer the relationship. It is reasonable to use the local coassociation coefficient between the samples in unstable set and the discovered core regions to obtain the fringe region of each cluster. For each sample $x \in \mathrm{US}(V)$, we calculate the local co-association coefficient $A_{l i}(x)$ between the sample and the core regions $\operatorname{Co}\left(C_{i}\right),(i=1,2, \ldots, k)$ and search the minimum value of the local co-association coefficient $A_{l i}(x),(i=1,2, \ldots, k)$. In order to reduce the decision risk, we use the following method to get the fringe region of each cluster.

Suppose

$$
A_{l j^{*}}=\min _{1 \leq i \leq k} A_{l i}(x)
$$

and

$$
T=\left\{j: A_{l j}(x)-A_{l j^{*}} \leq \varepsilon, j \neq j^{*}\right\},
$$

where $\varepsilon$ is a given parameter.

$$
\begin{aligned}
& 1 \text { If } T \neq \phi \text {, then } x \in \operatorname{Fr}\left(C_{j}\right) \text { and } x \in \operatorname{Fr}\left(C_{j}^{*}\right) \text {. } \\
& 2 \text { If } T=\phi \text {, then } x \in \operatorname{Fr}\left(C_{j}^{*}\right) \text {. }
\end{aligned}
$$

Algorithm 3 is the process of finding the fringe region of each cluster.

According to the above strategy, a three-way clustering is naturally formed. Unlike existing algorithms that treat every sample equally in the construction of the underlying structure, 3WC-SSS uses the similarity in spectral clustering to represent the co-association frequency and get the stability of each sample by using the determinacy function. With this stability, the universe is divided into stable set and unstable set. These samples in the stable set represent a clear structure which is easy to be discovered. We use k-means algorithm to obtain the core region of each cluster. The samples in the unstable set are assigned to the fringe region of each cluster by local co-association coefficient corresponding to the discovered core regions. The whole process can be shown as Algorithm 4.

\section{Experimental Results}

In order to illustrate the effectiveness of our proposed $3 \mathrm{WC}$ SSS algorithm, Two synthetic datasets and five UCI [31] data sets are employed in this section. The details of these data sets are shown in Table 3 and Table 4, respectively.

In this paper, we use ACC, FMIARI [32] and AS [33] , four quantitative indices to evaluate the performances of 


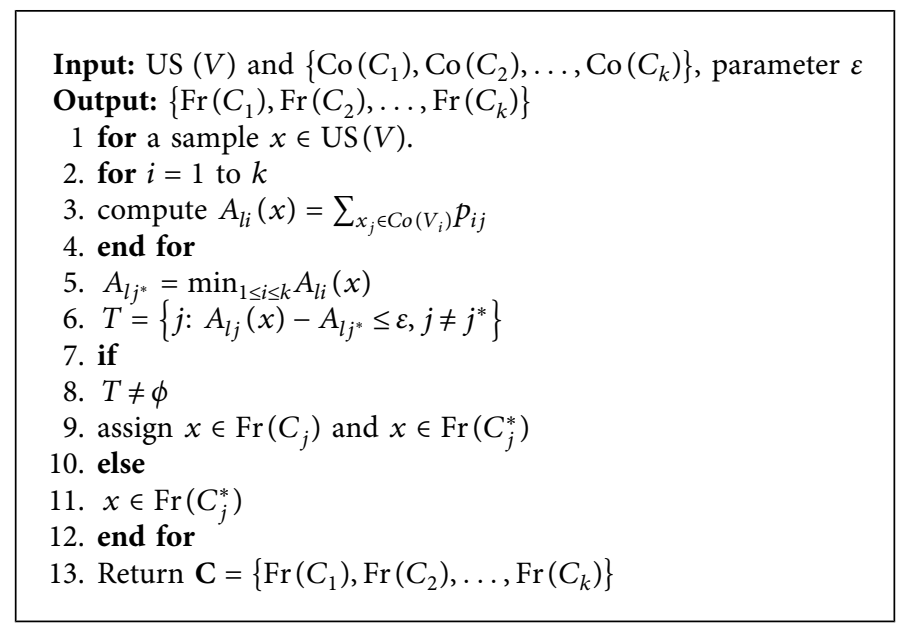

Algorithm 3: Find the fringe region of each cluster

Input: $X=\left\{x_{1}, \ldots, x_{n}\right\}$, the size of neighbour $K$, the number of cluster $k$, parameters $\sigma$ and $\varepsilon$.

Output: three-way clustering $\mathbb{C}=\left\{\left(\operatorname{Co}\left(C_{1}\right), \operatorname{Fr}\left(C_{1}\right)\right),\left(\operatorname{Co}\left(C_{2}\right), \operatorname{Fr}\left(C_{2}\right)\right), \ldots,\left(\operatorname{Co}\left(C_{k}\right), \operatorname{Fr}\left(C_{k}\right)\right)\right\}$

1. $\left\{\operatorname{Co}\left(C_{1}\right), \operatorname{Co}\left(C_{2}\right), \ldots, \operatorname{Co}\left(C_{k}\right)\right\} \leftarrow$ Algorithm 2

2. $\left\{\operatorname{Fr}\left(C_{1}\right), \operatorname{Fr}\left(C_{2}\right), \ldots, \operatorname{Fr}\left(C_{k}\right)\right\} \leftarrow$ Algorithm 3

3. Return $\mathbb{C}=\left\{\left(\operatorname{Co}\left(C_{1}\right), \operatorname{Fr}\left(C_{1}\right)\right),\left(\operatorname{Co}\left(C_{2}\right), \operatorname{Fr}\left(C_{2}\right)\right), \ldots,\left(\operatorname{Co}\left(C_{k}\right), \operatorname{Fr}\left(C_{k}\right)\right)\right\}$

Algorithm 4: 3WC-SSS algorithm

TABLE 3: synthetic data sets

\begin{tabular}{lccc}
\hline Data sets & Samples & Attributes & Classes \\
\hline A1 & 3000 & 2 & 20 \\
S1 & 5000 & 2 & 15 \\
\hline
\end{tabular}

TABLE 4: UCI data sets

\begin{tabular}{lccc}
\hline Data sets & Samples & Attributes & Classes \\
\hline Zoo & 101 & 18 & 7 \\
Iris & 150 & 4 & 3 \\
Wine & 178 & 13 & 3 \\
Dermatology & 366 & 33 & 6 \\
Segmentation & 2310 & 19 & 7 \\
\hline
\end{tabular}

clustering algorithms. The range of the ACC, FMI, AS, ARI is between 0 and 1 . The higher value indicates a better clustering result. Because all the above evaluation indices are only adapted to hard clustering algorithm, for fair comparison on clustering results with two kinds of representation, we take core region of each cluster obtained by 3WCSSS as the single set to compare with other algorithms. For comparing the clustering effect, the performance of 3WCSSS is compared with the performances of kmeans and FCM [9]. All experiments are repeated 10 times and we adopt the average result for 10 times as the final valid resultsTable 5 in Table 6 this Table 7 paper. Tables 8 show the clustering evaluation on 7 datasets. The best performances for each dataset are highlighted in bold.

Following experimental performances in Tables 6-8, it is not difficult to find that our proposed 3TW-SSS achieves the highest results of FMI, ARI, AS on all 7 datasets. The results
TABLE 5: Performances of ACC

\begin{tabular}{lccc}
\hline Data sets & kmeans & FCM & 3WC-SSS \\
\hline A1 & 0.9656 & 0.9711 & $\mathbf{0 . 9 7 7 2}$ \\
S1 & 0.9667 & 0.9718 & $\mathbf{0 . 9 7 9 2}$ \\
Zoo & 0.8881 & $\mathbf{0 . 9 1 9 9}$ & 0.9011 \\
Iris & 0.7183 & 0.7453 & $\mathbf{0 . 7 8 0 1}$ \\
Wine & 0.9550 & $\mathbf{0 . 9 7 8 9}$ & 0.9719 \\
Dermatology & 0.9899 & 0.9966 & $\mathbf{0 . 9 9 9 1}$ \\
Segmentation & 0.6563 & $\mathbf{0 . 7 1 3 4}$ & 0.6915 \\
\hline
\end{tabular}

TABle 6: Performances of FMI

\begin{tabular}{lccc}
\hline Data sets & kmeans & FCM & 3WC-SSS \\
\hline A1 & 0.9214 & 0.9321 & $\mathbf{0 . 9 4 5 3}$ \\
S1 & 0.9333 & 0.9514 & $\mathbf{0 . 9 6 0 5}$ \\
Zoo & 0.8221 & 0.8551 & $\mathbf{0 . 8 5 5 6}$ \\
Iris & 0.8185 & 0.8456 & $\mathbf{0 . 8 8 1 9}$ \\
Wine & 0.9253 & 0.9388 & $\mathbf{0 . 9 4 3 5}$ \\
Dermatology & 0.8891 & 0.8921 & $\mathbf{0 . 9 0 1 5}$ \\
Segmentation & 0.5864 & 0.6372 & $\mathbf{0 . 6 5 8 9}$ \\
\hline
\end{tabular}

show that the samples in core regions can improve the structure of the clustering results. This is because the samples in the core regions have higher stabilities than the samples in the fringe regions and we do not consider the samples in the fringe regions for computing FMI, ARI, AS.

Through observing Tables 5, we can find the performances of ACC that achieved by 3TW-SSS is a little lower than the results of other algorithm on some datesets. Such result indicates that though the samples in the core regions have higher stabilities, the results of accuracy may not be 
TABle 7: Performances of ARI

\begin{tabular}{lccc}
\hline Data sets & kmeans & FCM & 3WC-SSS \\
\hline A1 & 0.9111 & 0.9123 & $\mathbf{0 . 9 4 2 5}$ \\
S1 & 0.9437 & 0.9488 & $\mathbf{0 . 9 5 7 7}$ \\
Zoo & 0.8000 & 0.8101 & $\mathbf{0 . 8 1 2 7}$ \\
Iris & 0.8145 & 0.8222 & $\mathbf{0 . 8 2 3 8}$ \\
Wine & 0.9005 & 0.9116 & $\mathbf{0 . 9 1 4 9}$ \\
Dermatology & 0.8445 & 0.8563 & $\mathbf{0 . 8 6 8 2}$ \\
Segmentation & 0.5668 & 0.5888 & $\mathbf{0 . 6 0 0 6}$ \\
\hline
\end{tabular}

TABle 8: Performances of AS

\begin{tabular}{lccc}
\hline Data sets & kmeans & FCM & 3WC-SSS \\
\hline A1 & 0.8123 & 0.8224 & $\mathbf{0 . 8 3 2 8}$ \\
S1 & 0.8667 & 0.8674 & $\mathbf{0 . 8 6 7 7}$ \\
Zoo & 0.7442 & 0.7501 & $\mathbf{0 . 7 6 6 3}$ \\
Iris & 0.6891 & 0.7132 & $\mathbf{0 . 7 4 0 5}$ \\
Wine & 0.4763 & 0.5011 & $\mathbf{0 . 5 1 2 1}$ \\
Dermatology & 0.5113 & 0.5612 & $\mathbf{0 . 5 6 5 5}$ \\
Segmentation & 0.4589 & 0.5180 & $\mathbf{0 . 5 4 2 1}$ \\
\hline
\end{tabular}

better because the denominator $n$ become smaller when we compute ACC by deleting the samples in the fringe regions.

According to the experimental performances, we can find the samples with higher stabilities can provide more instructive information for detecting the pre-structure of data set and our proposed method for finding the core region is effective.

\section{Concluding Remarks}

In this paper, we develop a new three-way clustering algorithm, namely, 3W-SSS, by integrating similarity-based sample's stability into the idea of three-way clustering. In the proposed theory, the similarity of two samples is used to define the frequencies of two samples and the samples stability is calculated based on the defined frequencies and determinacy function. With this stability, the universe is divided into stable set and unstable set. The samples in the stable set are assigned into the core region of each cluster by using traditional clustering algorithm. The samples in the unstable set are assigned into the fringe region of corresponding cluster according to distances between the elements and the center of the cluster core regions. One contribution of $3 \mathrm{~W}$-SSS is that this algorithm can discovers a structure based on stable samples and then gradually assigns unstable samples to the pre-discovered structure. The experimental results on several datasets show that 3TW-SSS can significantly improve the structure of clustering results. The following problems may deserve further investigation.

1 For the sake of simplicity, the number of clusters $k$ is known in this paper. However, how to choose $k$ and determine the number of clusters can be researchedlenleadertwodots

2 Some other strategies for co-association frequency and determinacy function can be considered.

\section{Data Availability}

The data used to support the findings of this study are available from the corresponding author upon request.

\section{Conflicts of Interest}

The authors declare no conflict of interest.

\section{Acknowledgments}

This work was supported in part by National Natural Science Foundation of China (Nos. 62076111 and 61773012), Natural Science Foundation of the Jiangsu Higher Education Institutions of China (No. 15KJB110004), and Postgraduate Research \& Practice Innovation Program of Jiangsu Province.

\section{References}

[1] R. Xu and D. WunschII, "Survey of clustering algorithms," IEEE Transactions on Neural Networks, vol. 16, no. 3, pp. 645-678, 2005.

[2] A. Onan, "A fuzzy-rough nearest neighbor classifier combined with consistency-based subset evaluation and instance selection for automated diagnosis of breast cancer," Expert Systems with Applications, vol. 42, no. 20, pp. 6844-6852, 2015.

[3] A. Onan, S. Korukoğlu, and H. Bulut, "A hybrid ensemble pruning approach based on consensus clustering and multiobjective evolutionary algorithm for sentiment classification," Information Processing \& Management, vol. 53, no. 4, pp. 814-833, 2017.

[4] H. Shi, P. X. Wang, X. B. Yang, and H. L. Yu, "An improved mean imputation clustering algorithm for incomplete data," Neural Processing Letters, 2021.

[5] A. K. Jain, M. N. Murty, and P. J. Flynn, "Data clustering," ACM Computing Surveys, vol. 31, no. 3, pp. 264-323, 1999.

[6] A. K. Jain, "Data clustering: 50 years beyond k-means," Pattern Recognition Letters, vol. 31, pp. 651-666, 2010.

[7] J. Macqueen, "Some methods for classification and analysis of multivariate observations," in Proceedings of 5-th Berkeley Symposium on Mathematical Statistics and Probabilityvol. 1, , pp. 281-197, University of California Press, 1967.

[8] M. Fiedler, "Algebraic connectivity of graphs," Czechoslovak Mathematical Journal, vol. 23, no. 2, pp. 298-305, 1973.

[9] J. Bezdek, Pattern Recognition with Fuzzy Objective Function Algorithms, Plenum Press, NewYork, NY, USA, 1981.

[10] P. Lingras and C. West, "Interval set clustering of web users with rough k-means," Journal of Intelligent Information Systems, vol. 23, no. 1, pp. 5-16, 2004.

[11] S. Mitra, H. Banka, and W. Pedrycz, "Rough-fuzzy collaborative clustering," IEEE Transactions on Systems, Man and Cybernetics, Part B (Cybernetics), vol. 36, no. 4, pp. 795-805, 2006.

[12] S. Mitra, W. Pedrycz, and B. Barman, "Shadowed c-means: integrating fuzzy and rough clustering," Pattern Recognition, vol. 43, no. 4, pp. 1282-1291, 2010. 
[13] Y. Yao, "An outline of a theory of three-way decisions," in Proceedings of the Rough Sets and Current Trends in Computing, pp. 1-17, Springer, Chengdu, China, August, 2012.

[14] Y. Yao, "Three-way decisions and cognitive computing," Cognitive Computation, vol. 8, no. 4, pp. 543-554, 2016.

[15] Z. Pawlak, "Rough sets," International Journal of Computer \& Information Sciences, vol. 11, pp. 314-356, 1982.

[16] L. A. Zadeh, "Fuzzy sets," Information and Control, vol. 8, no. 3, pp. 338-353, 1965.

[17] W. Pedrycz, "Shadowed sets: representing and processing fuzzy sets," IEEE Transactions on Systems, Man and Cybernetics, Part B (Cybernetics), vol. 28, no. 1, pp. 103-109, 1998.

[18] H. Yu, "A framework of three-way cluster analysis," in Proceedings of the International Joint Conference on Rough Sets, pp. 300-312, Olsztyn, Poland, July, 2017.

[19] H. Yu, P. Jiao, Y. Yao, and G. Wang, "Detecting and refining overlapping regions in complex networks with three-way decisions," Information Sciences, vol. 373, pp. 21-41, 2016.

[20] H. Yu, C. Zhang, and G. Wang, "A tree-based incremental overlapping clustering method using the three-way decision theory," Knowledge-Based Systems, vol. 91, pp. 189-203, 2016.

[21] M. K. Afridi, N. Azam, J. Yao, and E. Alanazi, "A three-way clustering approach for handling missing data using gtrs," International Journal of Approximate Reasoning, vol. 98, pp. 11-24, 2018.

[22] P. Wang and Y. Yao, "Ce3: a three-way clustering method based on mathematical morphology," Knowledge-Based Systems, vol. 155, pp. 54-65, 2018.

[23] H. Yu, X. Wang, G. Wang, and X. Zeng, "An active three-way clustering method via low-rank matrices for multi-view data," Information Sciences, vol. 507, pp. 823-839, 2020.

[24] P. Wang, H. Shi, X. Yang, and J. Mi, "Three-way k-means: integrating k-means and three-way decision," International Journal of Machine Learning and Cybernetics, vol. 10, no. 10, pp. 2767-2777, 2019.

[25] P. Wang and X. Chen, "Three-way ensemble clustering for incomplete data," IEEE Access, vol. 8, Article ID 91855, 2020.

[26] H. Yu, Z. Chang, G. Wang, and X. Chen, "An efficient threeway clustering algorithm based on gravitational search," International Journal of Machine Learning and Cybernetics, vol. 11, no. 5, pp. 1003-1016, 2020.

[27] P. Wang and X. Yang, "Three-way clustering method based on stability theory," IEEE Access, vol. 9, Article ID 33944, 2021.

[28] F. Li, Y. Qian, J. Wang, C. Dang, and L. Jing, "Clustering ensemble based on sample's stability," Artificial Intelligence, vol. 273, pp. 37-55, 2019.

[29] N. Otsu, "A threshold selection method from gray-level histograms," IEEE Transactions on Systems, Man, and Cybernetics, vol. 9, no. 1, pp. 62-66, 1979.

[30] U. von Luxburg, "A tutorial on spectral clustering," Statistics and Computing, vol. 17, no. 4, pp. 395-416, 2007.

[31] C. L. Blake and C. J. Merz, "UCI Machine Learning Repository," 2005, http://www.ics.uci.edu/mlearn/MLRepository. html.

[32] A. Fahad, N. Alshatri, Z. Tari et al., "A survey of clustering algorithms for big data: taxonomy and empirical analysis," IEEE Transactions on Emerging Topics in Computing, vol. 2, no. 3, pp. 267-279, 2014.

[33] P. J. Rousseeuw, "Silhouettes: a graphical aid to the interpretation and validation of cluster analysis," Journal of Computational and Applied Mathematics, vol. 20, pp. 53-65, 1987. 